\title{
Hemodynamic effects of ephedrine and phenylephrine bolus injection in patients in the prone position under general anesthesia for lumbar spinal surgery
}

\author{
JIANGYAN XIA ${ }^{1}$, YONGYING SUN ${ }^{2}$, JING YUAN $^{1}$, XINJIAN LU $^{1}$, ZHENDAN PENG $^{1}$ and NING YIN ${ }^{1}$ \\ Departments of ${ }^{1}$ Anesthesiology and ${ }^{2}$ Stomatology, Zhongda Hospital, \\ Southeast University, Nanjing, Jiangsu 210009, P.R. China
}

Received November 28, 2014; Accepted December 14, 2015

DOI: $10.3892 / \mathrm{etm} .2016 .3432$

\begin{abstract}
Ephedrine and phenylephrine (PE) are vasoconstrictors commonly used to restore the blood pressure (BP) to normal values. The aim of the present study was to investigate the effects of ephedrine and PE bolus administration on intra-arterial systolic BP (ISBP), intra-arterial diastolic BP (IDBP) and cardiac output (CO) in patients undergoing lumbar spine surgery in the prone position under general anesthesia (GA). In this prospective, randomized, and double-blind study, a total of 60 patients aged 20-60 years and undergoing elective lumbar spine surgery were administered either a single dose of ephedrine $(0.1 \mathrm{mg} / \mathrm{kg})$ or PE $(1 \mu \mathrm{g} / \mathrm{kg})$ through a central venous catheter as a bolus injection following the achievement of a stable hemodynamic status for $\geq 10 \mathrm{~min}$. Following bolus injection of ephedrine or PE, a significant increase in ISBP was observed in the two experimental groups compared with pre-ephedrine and pre-PE values. The duration of the increment in ISBP however was significantly longer in the ephedrine group compared with the PE group. A similar response was observed in IDBP. A significant increase in CO began 1 min following ephedrine injection and lasted for the entire observation period, whereas the increase was only sustained for 3 min following bolus injection in the PE group. The results of the present study demonstrated that bolus ephedrine produces a more persistent pressor response and durable increase in $\mathrm{CO}$ and CI compared with PE when patients are in the prone position with GA for spine surgery.
\end{abstract}

Correspondence to: Dr Jiangyan Xia and Dr Ning Yin, Department of Anesthesiology, Zhongda Hospital, Southeast University, 87 DingJiaQiao Road, Nanjing, Jiangsu 210009, P.R. China

E-mail: jiangyanxia731026@aliyun.com

E-mail: yinning882000@126.com

Key words: ephedrine, phenylephrine, prone position, general anesthesia, hemodynamic

\section{Introduction}

Surgical procedures on patients in the prone position while under general anesthesia (GA) may be associated with significant hemodynamic changes including reductions in cardiac index (CI) and arterial blood pressure (BP), as well as increases in central venous pressure (CVP) and systemic vascular resistance (SVR). These changes may be attributed to alteration of intra-abdominal pressure and compression of the thoracic space (1-4). Intra-operative hypotension (IOH) has been reported to be independently associated with adverse perioperative outcomes and associated with patient postoperative long-term mortality (5-7). Perioperative stroke has often been attributed to $\mathrm{IOH}(8)$, and $\mathrm{IOH}$ has been reported to be one of the most important concerns associated with the occurrence of postoperative myocardial ischemia and infarction (6). It is crucial to avoid the occurrence or shorten the duration of IOH and subsequently prevent peri-operative complications. Clinicians usually reverse $\mathrm{IOH}$ by decreasing anesthetic dosages, enhancing intravenous fluid administration or applying vasopressors (9).

Ephedrine and phenylephrine (PE) are two commonly used vasoconstrictors that restore BP to normal values $(10,11)$. Ephedrine acts on $\alpha$ - and $\beta$-adrenergic receptors and displaces norepinephrine from adrenergic terminals (12-14). The release of catecholamines and subsequent activation of adrenergic receptors is thought to be the primary mechanism underlying the cardiovascular response to ephedrine. PE predominantly acts on the $\alpha 1$-adrenergic receptor (12-14), which causes a direct increase in SVR, arterial pressure and left ventricular afterload.

A previous study demonstrated that cerebral tissue oxygen saturation $\left(\mathrm{SctO}_{2}\right)$ was significantly decreased following PE administration, but not following ephedrine treatment, and cardiac output (CO) was identified to have the most significant association with $\mathrm{SctO}_{2}(15)$. The use of PE to correct hypotension induced by anesthesia has a negative impact on $\mathrm{SctO}_{2}$, whereas ephedrine maintains frontal lobe oxygenation, which may be associated with an increase in CO (14). Bolus PE reduced maternal CO when compared with ephedrine during elective spinal anesthesia in cesarean delivery (12). 
The hemodynamic effects of ephedrine and PE used while patients are in the prone position under GA have not been fully described. The aim of the present study was to investigate the effects of ephedrine and PE bolus administration on physiological variables including intra-arterial systolic BP (ISBP), intra-arterial diastolic BP (IDBP), CO, heart rate (HR), stroke volume variation (SVV), cardiac index (CI), stroke volume (SV), SV index (SVI), central venous pressure (CVP), and bispectral index (BIS) of anesthetized patients in the prone position.

\section{Materials and methods}

Patients, study design and ethical considerations. This was a prospective, randomized, and double-blind study that was performed at Zhongda Hospital, Southeast University (Nanjing, China). A total of 60 patients were randomly assigned to one of two groups: An ephedrine group $(n=30)$, and a PE group $(n=30)$. Patients included in the study were aged between 20 and 60 years, were undergoing elective lumbar spine surgery, and had an American Society of Anesthesiologists physical status of I-II (16). Patients were excluded from this study if they had a history of symptomatic cardiovascular disorders including sinus bradycardia, hypertension, diabetes mellitus, a body mass index $>28 \mathrm{~kg} / \mathrm{m}^{2}$, or other disorders known to affect autonomic nervous activity.

Over the course of the study, if the ISBP of a patient increased to $>160 \mathrm{mmHg}$ or the IDBP increased to $>95 \mathrm{mmHg}$ for $>2 \mathrm{~min}$ following ephedrine $(0.1 \mathrm{mg} / \mathrm{kg})$ or PE administration, the patient was withdrawn from the study. Patients were also withdrawn from the study if their HR decreased to $<45$ beats $/ \mathrm{min}$ and required a bolus dose of 25-50 $\mu \mathrm{g}$ nitroglycerin, 20-40 mg esmolol or $0.5 \mathrm{mg}$ atropine as a rescue treatment to recover BP or HR. Shanghai, China

The study protocol was approved by the Institutional Review Board of Zhongda Hospital, Southeast University. Patients provided both verbal consent and signed informed consent forms. The study was registered on the Chinese Clinical Trial Center (ChiCTR; Registration no. ChiCTR-RNRC-13003094; Registration date, March 7 2013; Principal investigator, Dr Jiangyan Xia).

Study protocol. Following patient anesthesia and endotracheal intubation, a radical intra-arterial catheter and a central venous catheter with BIS electrodes were inserted in addition to measurement of vital signs. Lead II electrocardiography data were continuously monitored throughout the study. A third generation Vigileo $^{\mathrm{TM}} /$ FloTrac $^{\mathrm{TM}}$ device (Edwards Lifesciences, Irvine, CA, USA) was used for the continuous recording of hemodynamic parameters.

All patients were pre-oxygenated with $100 \%$ oxygen via a face mask for 2-3 min prior to the induction of GA. The protocol of induction and maintenance of GA were identical between the two groups. Briefly, GA was induced with fentanyl (2-3 $\mu \mathrm{g} / \mathrm{kg}$; Yichang Humanwell Pharmaceutical, Co., Ltd., Yichang, China), followed by the intravenous administration of propofol (2-2.5 mg/kg; AstraZeneca plc, Wuxi, China). Endotracheal intubation was facilitated with intravenous cisatracurium $(0.15 \mathrm{mg} / \mathrm{kg}$; Jiangsu Hengrui Medicine, Co., Ltd., Lianyungang, China). Anesthesia was maintained with a 1.5-3\% end-tidal concentration of sevoflurane (Maruishi Pharmaceutical Co., Ltd., Osaka, Japan) 50:50 oxygen and air mixture, remifentanyl (5-10 $\mu \mathrm{g} / \mathrm{kg} / \mathrm{h}$; Yichang Humanwell Pharmaceutical, Co., Ltd.), and cisatracurium (150 $\mu \mathrm{g} / \mathrm{kg} / \mathrm{h})$. The concentration of inhaled sevoflurane was adjusted to maintain the BIS between 40-60. Ventilation was controlled using a mechanical ventilator. The tidal volume was held constant at $10 \mathrm{ml} / \mathrm{kg}$ and end-tidal $\mathrm{CO}_{2}$ was maintained at $30-40 \mathrm{mmHg}$ by adjusting the frequency of ventilation. The patients were placed in the prone position following induction of anesthesia and confirmation of the correct position of the endotracheal tube. Hetastarch $(10 \mathrm{ml} / \mathrm{kg}$; 6\%; Jiangsu Hengrui Medicine, Co., Ltd.) was administered as a bolus fluid expansion for $30 \mathrm{~min}$, prior to the administration of $15 \mathrm{ml} / \mathrm{kg} / \mathrm{h}$ lactated Ringer's solution (Chimin Pharmaceutical, Co., Ltd., Zhejiang, China).

A single dose of ephedrine $(0.1 \mathrm{mg} / \mathrm{kg}$; Northeast Pharmaceutical Group, Co., Ltd., Shenyang, China) or PE (1 $\mu \mathrm{g} / \mathrm{kg}$; Shanghai Harvest Pharmaceutical, Co.,Ltd., Shanghai, China) was administered through the central venous catheter as a bolus injection after the patient achieved a stable hemodynamic status for $\geq 10 \mathrm{~min}$. The ISBP was maintained at $85-105 \mathrm{mmHg}$ by adjusting the concentration of end-tidal sevoflurane. Ephedrine was diluted to $5 \mathrm{mg} / \mathrm{ml}(0.02 \mathrm{ml} / \mathrm{kg})$ with saline, and $\mathrm{PE}$ was diluted to $50 \mu \mathrm{g} / \mathrm{ml}(0.02 \mathrm{ml} / \mathrm{kg})$. Hemodynamic parameters were measured and recorded at $1 \mathrm{~min}$ intervals for $10 \mathrm{~min}$ (T1-T10) following bolus injection of ephedrine or PE. All measurements were performed during the surgical procedure under GA in the prone position. No vasoconstrictors or vasodilators were used within $1 \mathrm{~h}$ prior to hemodynamic measurements and the concentrations of the general anesthetics used to maintain the anesthesia were not changed for $\geq 10 \mathrm{~min}$ prior to the measurements. There was also no significant blood loss $(<200 \mathrm{ml})$ resulting from the surgical procedure prior to administration of the bolus ephedrine or PE.

Assessments. The primary parameters assessed in this study were ISBP, IDBP and CO. Secondary parameters included CVP, CI, SVV, SVR and HR. Baseline values (immediately prior to ephedrine or PE administration) and post-ephedrine/PE values were measured and compared. Adverse events were also recorded.

Statistical analysis. Statistical analysis and calculations were conducted using GraphPad Prism 5.0 (GraphPad Software, Inc., La Jolla, CA, USA). Data were expressed as mean \pm standard deviation. Intra-arterial mean BP (IMAP) was calculated as IDBP + 1/3 x (ISBP - IDBP). Intergroup statistical comparisons were performed using two-way analysis of variance (ANOVA), followed by Student's t-test with Bonferroni's correction. BP and HR values over time following ephedrine or PE injection were analyzed using repeated one-way ANOVA, followed by a paired t-test with Bonferroni's correction for paired data in each group. $\mathrm{P}<0.05$ was considered to indicate a statistically significant result.

\section{Results}

Demographic and baseline characteristics. The two experimental groups were comparable with respect to age, gender, 
Table I. Baseline data and hemodynamic parameters in the two groups at 0 min (mean \pm standard deviation).

\begin{tabular}{|c|c|c|c|}
\hline Characteristic & Ephedrine & Phenylephrine & P-value \\
\hline Gender (male/female) & $17 / 13$ & $14 / 16$ & 0.3006 \\
\hline Age (years) & $52.2 \pm 4.27$ & $51.4 \pm 5.25$ & 0.5028 \\
\hline Weight (kg) & $66.7 \pm 9.93$ & $67.3 \pm 10.14$ & 0.8178 \\
\hline Height $(\mathrm{cm})$ & $166.0 \pm 8.54$ & $166.6 \pm 7.43$ & 0.7727 \\
\hline \multicolumn{4}{|c|}{ Hemodynamic parameters prior to anesthesia } \\
\hline $\mathrm{SBP}(\mathrm{mmHg})$ & $131.5 \pm 11.0$ & $134.5 \pm 12.2$ & 0.3117 \\
\hline $\mathrm{DBP}(\mathrm{mmHg})$ & $78.8 \pm 7.6$ & $81.6 \pm 7.9$ & 0.1672 \\
\hline HR (beats/min) & $71.3 \pm 7.7$ & $73.5 \pm 8.6$ & 0.2919 \\
\hline \multicolumn{4}{|c|}{ Hemodynamic parameters prior to bolus injection } \\
\hline ISBP $(\mathrm{mmHg})$ & $95.8 \pm 6.7$ & $96.4 \pm 7.6$ & 0.7744 \\
\hline IDBP (mmHg) & $54.5 \pm 5.8$ & $54.4 \pm 5.6$ & 1.0000 \\
\hline IMAP (mmHg) & $68.2 \pm 5.7$ & $68.4 \pm 5.8$ & 0.9044 \\
\hline HR (beats/min) & $61.2 \pm 7.2$ & $61.0 \pm 6.3$ & 0.8649 \\
\hline $\mathrm{CO}(1 / \mathrm{min})$ & $3.7 \pm 0.8$ & $3.7 \pm 0.8$ & 0.8921 \\
\hline $\mathrm{CI}\left(1 / \mathrm{min} / \mathrm{m}^{2}\right)$ & $2.2 \pm 0.4$ & $2.2 \pm 0.4$ & 0.8427 \\
\hline $\mathrm{CVP}(\mathrm{mmHg})$ & $7.4 \pm 2.6$ & $7.3 \pm 2.6$ & 0.8435 \\
\hline BIS & $41.1 \pm 3.7$ & $42.7 \pm 3.5$ & 0.1194 \\
\hline SVV & $10.7 \pm 2.6$ & $10.2 \pm 2.1$ & 0.4555 \\
\hline SV & $60.3 \pm 8.1$ & $60.2 \pm 9.6$ & 0.9652 \\
\hline \multicolumn{4}{|l|}{ End-tidal concentration } \\
\hline Sevoflurane (\%) & $1.94 \pm 0.53$ & $1.91 \pm 0.54$ & 0.8087 \\
\hline Remifentanyl (mg/h) & $0.34 \pm 0.07$ & $0.34 \pm 0.08$ & 0.2429 \\
\hline
\end{tabular}

SBP, systolic blood pressure; DBP, diastolic blood pressure; HR, heart rate; ISBP, intra-arterial SBP; IDBP, intra-arterial DBP; IMAP, intra-arterial mean blood pressure; CO, cardiac output; CI, cardiac index, CVP, central venous pressure; BIS, bispectral index; SVV, stroke volume variation; $\mathrm{SV}$, stroke volume.

body weight and height $(\mathrm{P}>0.05$; Table I). There was also no significant difference between the two experimental groups with regard to the end-tidal concentration of sevoflurane and remifentanyl used to maintain the depth of anesthesia (DOA; $\mathrm{P}>0.05$; Table I). Baseline (0 min, immediately prior to ephedrine or PE injection) hemodynamic parameters of ISBP, IDBP, IMAP, CO, SVV, CVP, CI, SVR and HR were comparable between the two experimental groups $(\mathrm{P}>0.05$; Table I). All 60 patients completed the study and were included in the statistical analysis. No patients were excluded from the study due to exaggerated pressor reaction or severe tachycardia.

Hemodynamic changes. Following the bolus injection of ephedrine or PE, a significant increase in ISBP was observed in the two experimental groups compared with pre-ephedrine and pre-PE values (Fig. 1A). The duration of the increment in ISBP was considerably longer in the ephedrine group compared with the PE group. A similar response was observed in IDBP (Fig. 1B) and IMAP (Fig. 1C). Ephedrine caused a significant increase in the HR from 1 to $10 \mathrm{~min}(61.27$ to 70.20 beats/min), whereas PE only caused a 2 -min reduction in the HR (1-2 min; 60.97 to 56.87 beats/min; Fig. 1D). Intravenous ephedrine resulted in a decline in CVP, whereas $\mathrm{PE}$ produced a significant increase in CVP at 2-3 min (Fig. $1 E)$. The significant elevation in ISBP, IDBP and IMAP was initiated at 1 min following bolus injection in the ephedrine group, reached maximum values at $3 \mathrm{~min}$ and lasted for $10 \mathrm{~min}$. In the PE group, the effect only lasted 6 min and the maximum was reached $1 \mathrm{~min}$ following treatment. A significant increase in $\mathrm{CO}$ and $\mathrm{CI}$ (Fig. $1 \mathrm{~F}$ and G) was initiated at 1 min after dosing and lasted for the entire observation period following ephedrine injection, whereas it was only sustained for $3 \mathrm{~min}$ after the bolus injection of PE. The significant increase in SV (Fig. 1H) lasted from 2 to $8 \mathrm{~min}$ and 1 to $4 \mathrm{~min}$ in the ephedrine and PE groups, respectively. Treatment with either bolus ephedrine or PE caused an instant and transient elevation and then a subsequent significant reduction in SVV (Fig. 1I). The significant reduction in SVV lasted longer in the ephedrine group (from 2 to $10 \mathrm{~min}$ ) compared with the PE group (from 2 to $3 \mathrm{~min}$ ).

Safety assessments. In the present study, ephedrine and PE were used at doses that are comparatively low for a clinical setting in order to observe the pressor response and hemodynamic effects during GA in the prone position. Intervention was only provided when patient ISBP was $<105 \mathrm{mmHg}$ or the IMAP $\leq 20 \%$ compared with the pre-induction values. No patient appeared to have an exaggerated pressor response or severe tachycardia following the intravenous administration of $0.1 \mathrm{mg} / \mathrm{kg}$ ephedrine or $1 \mu \mathrm{g} / \mathrm{kg}$ PE. 


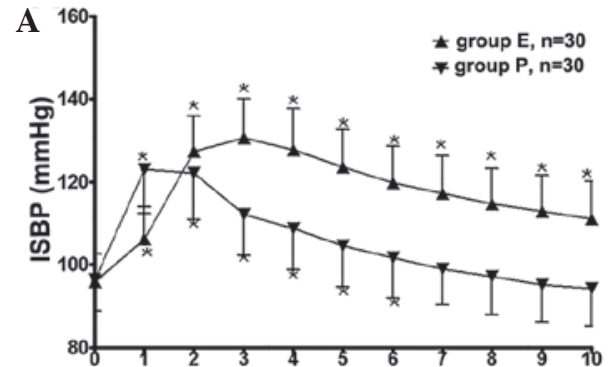

Time after intravenous ephedrine (min)

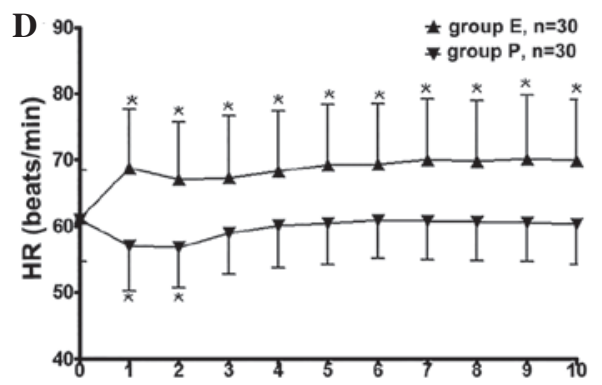

Time after intravenous ephedrine ( $\mathrm{min})$

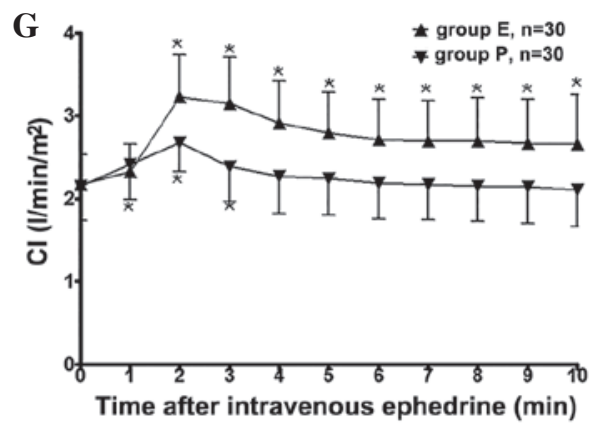

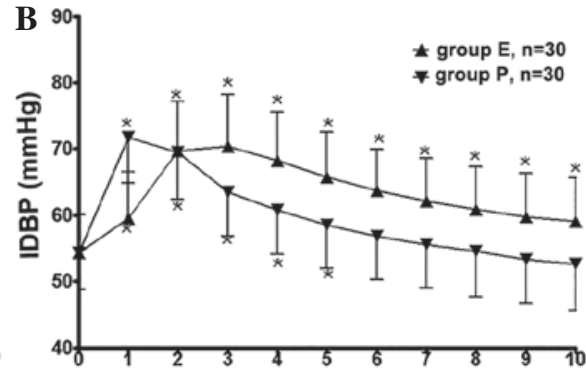

Time after intravenous ephedrine $(\mathrm{min})$

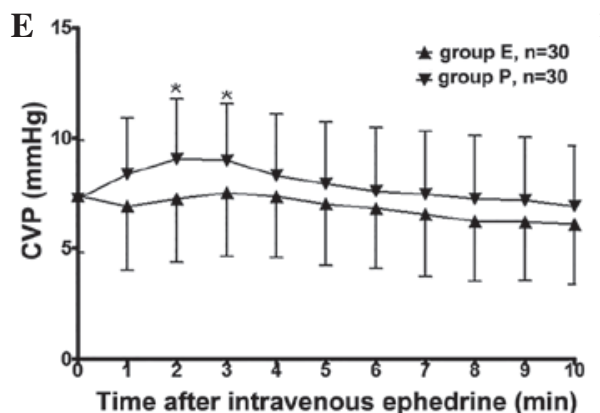

Time after intravenous ephedrine (min)

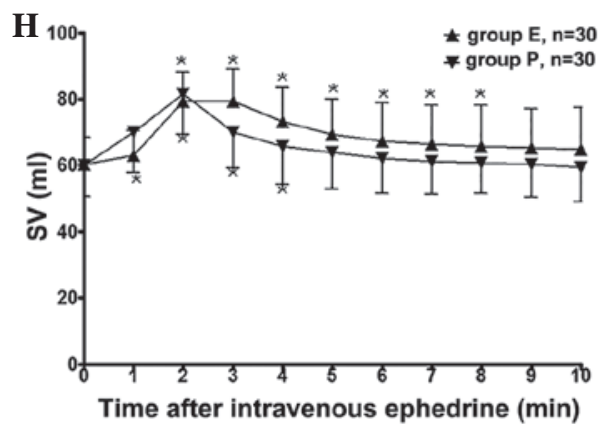

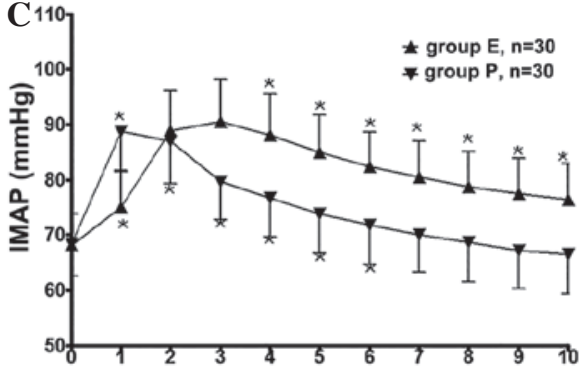

Time after intravenous ephedrine ( $\mathrm{min})$

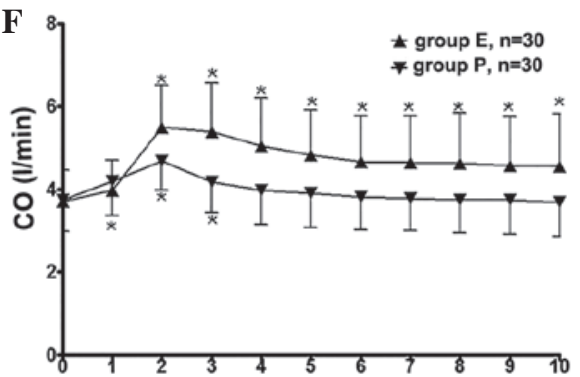

Time after intravenous ephedrine ( $\mathrm{min})$

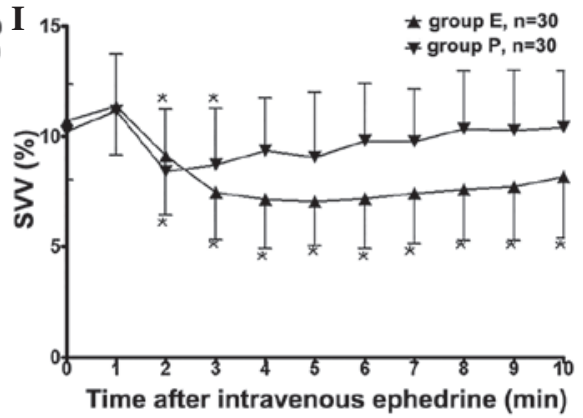

Figure 1. Hemodynamic parameters of patients that received a single dose of $\mathrm{E}(0.1 \mathrm{mg} / \mathrm{kg})$ or P $(1 \mu \mathrm{g} / \mathrm{kg})$ through a central venous catheter via bolus injection for the time period of 0 to $10 \mathrm{~min}$. The following parameters were measured: (A) ISBP, (B) IDBP, (C) IMAP, (D) HR, (E) CVP, (F) CO, (G) CI, (H) SV and (I) SVV. Data are presented as the mean \pm standard deviation. ${ }^{*} \mathrm{P}<0.05$ between the two groups. Group $\mathrm{P}$, patients treated with phenylephrine; group E, patients treated with ephedrine. ISBP, intra-arterial systolic blood pressure; IDBP, intra-arterial diastolic blood pressure; IMAP, intra-arterial mean blood pressure; HR, heart rate; CVP, central venous pressure; CO, cardiac output; CI, cardiac index; SV, stroke volume; SVV, stroke volume variation.

\section{Discussion}

Hypotension is a common complication observed following the use of various anesthesia techniques $(11,17)$, particularly in patients who are in the prone position while under GA $(1,4)$. $\mathrm{IOH}$ and intraoperative hemodynamic fluctuation contribute to perioperative morbidity and mortality $(6,7,18,19)$. Hypotension while prone may also be a risk factor for perioperative ischemic optic neuropathy in spine surgery (20). Avoiding IOH, or shortening the duration of $\mathrm{IOH}$ and maintaining stable vital parameters may provide patients with improved outcomes. Although vasoconstrictors have a rapid time of onset and reliable pressor effects, their inappropriate administration may lead to adverse outcomes such as tachycardia, hypertension and increased blood loss (21). Among all the frequently used vasopressors, ephedrine and PE are used to reverse or avoid hypotension associated with the performance of anesthesia techniques or application of anesthetic agents $(10,22,23)$.

The results of the present study suggested that compared with PE, a bolus intravenous injection of ephedrine resulted in a more durable pressor effect when patients were in the prone position under GA. This effect was accompanied by increased CO, CI, SV, SVI and HR, and decreased CVP and SVV, suggesting that the pressor response of ephedrine during GA in the prone position may be predominantly induced by the activation of $\beta$-adrenergic receptors. Compared with ephedrine, the hemodynamic changes induced by bolus PE include an acute but temporarily elevation of CO, CI, SV, SVI and CVP, and a short-term reduction in HR and SVV. There are two main mechanisms underlying the effects of PE on CO. PE increases ventricular afterload and thereby decreases SV and CO. Additionally, the release of blood from peripheral veins to central veins caused by PE elevates CVP and SV, and thus $\mathrm{CO}(24,25)$. It has been suggested that the impact of PE on $\mathrm{CO}$ is associated with preload dependency $(26,27)$. When the heart is preload-independent, a bolus injection of PE usually induces a reduction in $\mathrm{CO}$. When the heart is dependent on the blood volume that reaches the ventricle (preload dependent), PE boluses usually induce an increase in CO (28). Patients undergoing spinal surgery in the prone position may be preload-dependent due to the decreased returned blood volume resulting from enhanced intra-abdomen pressure and compression of the inferior vena cava and thoracic space. The bolus injection of PE would cause an instant increase in $\mathrm{CO}$ and $\mathrm{CI}$ and the increased venous returned blood would change the state of preload-dependent to preload-independent. The 
$\mathrm{CO}$ and $\mathrm{CI}$ would decline shortly following the transient elevation.

Controversy exists regarding the equivalent doses of $\mathrm{PE}$ and ephedrine. Previous studies have used ratios varying from 20:1 to $250: 1(12,29)$. In the patient population of the present study, a $0.1 \mu \mathrm{g} / \mathrm{kg}$ bolus of PE and a $0.1 \mathrm{mg} / \mathrm{kg}$ bolus of ephedrine restored the IMAP to within a range of $20 \%$ above or below baseline.

Previous investigations have demonstrated that the pressor effect of ephedrine is greater in anesthetized patients compared with awake patients (30). Propofol, enflurane and pre-medicated clonidine have been shown to enhance the pressor response to ephedrine (11,30-33). This response has been reported to be associated with differences in sympathetic tone and adrenergic receptor status (30-33). The pressor response has been found to be more intense at the lower basal level of sympathetic activity. Our previous study demonstrated that in comparison with the supine position, the prone position would induce a pressor response to ephedrine, which may be attributed to the enhanced parasympathetic nerve activity associated with the prone position (unpublished data).

Previous researchers postulated that the DOA may be one of the factors that influence the pressor response to vasoconstrictors $(30,32)$. However, in the present investigation, the two study groups followed the same anesthesia and fluid administration protocol. There was no significant difference between the dosages and concentrations of anesthetics used for induction and maintenance of anesthesia between the two groups. Pre-ephedrine and pre-PE hemodynamic parameters and BIS values were comparable between the two groups. Therefore, it was unlikely that there was a significant difference in the DOA between the two experimental groups.

One of the limitations of the present study was the use of a third generation Vigileo-FloTrac device for continuous recording of hemodynamic parameters. Although previous studies have verified the accuracy, sensitivity and reliability of this pressure-based cardiac output measurement system $(5,34)$, there have been reports that the pulse-contour method used by this system cannot accurately track changes in $\mathrm{CO}$ induced with PE and ephedrine $(35,36)$. Another limitation of the present study was that the autonomic nervous system (ANS) tone was not assessed during GA in the prone position, despite previous studies indicating that the tone of the ANS may influence the pressor response to some vasoconstrictors $(30,32)$. In addition, the level of cerebral tissue oxygenation and intraocular pressure were not monitored. Further studies are required to investigate the effects of vasopressors on the perfusion of tissues and major organs. Based on previous and present studies that have evaluated the inhibition or augmentation of biological responses to several vasoconstrictors under various anesthetic techniques, it can be derived that additional investigations focusing on amelioration of the hypo-reactivity and vasoplegia induced by sepsis through the regulation of the activity of ANS are required.

In conclusion, the present study demonstrated that bolus ephedrine will produce a more persistent pressor response and durable increase in $\mathrm{CO}$ and $\mathrm{CI}$ compared with PE when patients are in the prone position under GA for spine surgery. Ephedrine may be the more appropriate choice to reverse the $\mathrm{IOH}$ induced by GA in the prone position for spine surgery, as it induces a longer pressor response than PE. Ephedrine may also induce a more stable elevation of $\mathrm{CI}$ and $\mathrm{CO}$, which may be beneficial to the perfusion of major organs, particularly the cerebral nervous system.

\section{Acknowledgements}

The present study was supported by a grant from the Natural Science Foundation for Young Fund (grant no. $81201449 / \mathrm{H} 1502)$.

\section{References}

1. Poon KS, Wu KC, Chen CC, Fung ST, Lau AW, Huang CC and Wu RS: Hemodynamic changes during spinal surgery in the prone position. Acta Anaesthesiol Taiwan 46: 57-60, 2008.

2. Tabara Y, Tachibana-Iimori R, Yamamoto M, Abe M, Kondo I, Miki $\mathrm{T}$ and Kohara K: Hypotension associated with prone body position: A possible overlooked postural hypotension. Hypertens Res 28: 741-746, 2005.

3. Toyota S and Amaki Y: Hemodynamic evaluation of the prone position by transesophageal echocardiography. J Clin Anesth 10: 32-35, 1998 .

4. Dharmavaram S, Jellish WS, Nockels RP, Shea J, Mehmood R, Ghanayem A, Kleinman B and Jacobs W: Effect of prone positioning systems on hemodynamic and cardiac function during lumbar spine surgery: An echocardiographic study. Spine (Phila Pa 1976) 31: 1388-1393, 2006.

5. Biais M, Bernard O, Ha JC, Degryse C and Sztark F: Abilities of pulse pressure variations and stroke volume variations to predict fluid responsiveness in prone position during scoliosis surgery. Br J Anaesth 104: 407-413, 2010.

6. Bijker JB, Persoon S, Peelen LM, Moons KG, Kalkman CJ, Kappelle LJ and van Klei WA: Intraoperative hypotension and perioperative ischemic stroke after general surgery: A nested case-control study. Anesthesiology 116: 658-664, 2012.

7. Bijker JB, van Klei WA, Vergouwe Y, Eleveld DJ, van Wolfswinkel L, Moons KG and Kalkman CJ: Intraoperative hypotension and 1-year mortality after noncardiac surgery. Anesthesiology 111: 1217-1226, 2009.

8. Reich DL, Bodian CA, Krol M, Kuroda M, Osinski T and Thys DM: Intraoperative hemodynamic predictors of mortality, stroke and myocardial infarction after coronary artery bypass surgery. Anesth Analg 89: 814-822, 1999.

9. Hiltebrand LB, Koepfli E, Kimberger O, Sigurdsson GH and Brandt S: Hypotension during fluid-restricted abdominal surgery: Effects of norepinephrine treatment on regional and microcirculatory blood flow in the intestinal tract. Anesthesiology 114: 557-564, 2011.

10. Erkinaro T, Mäkikallio K, Kavasmaa T, Alahuhta S and Räsänen J: Effects of ephedrine and phenylephrine on uterine and placental circulations and fetal outcome following fetal hypoxaemia and epidural-induced hypotension in a sheep model. Br J Anaesth 93: 825-832, 2004.

11. Nazir I, Bhat MA, Qazi S, Buchh VN and Gurcoo SA: Comparison between phenylephrine and ephedrine in preventing hypotension during spinal anesthesia for cesarean section. J Obstet Anaesth Crit Care 2: 92-97, 2012.

12. Dyer RA, Reed AR, van Dyk D, Arcache MJ, Hodges O, Lombard CJ, Greenwood J and James MF: Hemodynamic effects of ephedrine, phenylephrine and the coadministration of phenylephrine with oxytocin during spinal anesthesia for elective cesarean delivery. Anesthesiology 111: 753-765, 2009.

13. Zaimis E: Vasopressor drugs and catecholamines. Anesthesiology 29: 732-762, 1968.

14. Nissen P, Brassard P, Jørgensen TB and Secher NH: Phenylephrine but not ephedrine reduces frontal lobe oxygenation following anesthesia-induced hypotension. Neurocrit Care 12: 17-23, 2010.

15. Meng L, Cannesson M, Alexander BS, Yu Z, Kain ZN, Cerussi AE, Tromberg BJ and Mantulin WW: Effect of phenylephrine and ephedrine bolus treatment on cerebral oxygenation in anaesthetized patients. Br J Anaesth 107: 209-217, 2011.

16. Daabiss M: American Society of Anesthesiologists physical status classification. Indian J Anaesth 55: 111-115, 2011. 
17. Reich DL, Hossain S, Krol M, Baez B, Patel P, Bernstein A and Bodian CA: Predictors of hypotension after induction of general anesthesia. Anesth Analg 101: 622-628, 2005.

18. Aronson S, Stafford-Smith M, Phillips-Bute B, Shaw A, Gaca J and Newman M; Cardiothoracic Anesthesiology Research Endeavors: Intraoperative systolic blood pressure variability predicts 30-day mortality in aortocoronary bypass surgery patients. Anesthesiology 113: 305-312, 2010.

19. Dwyer T, Ponsonby AL, Newman NM and Gibbons LE: Prospective cohort study of prone sleeping position and sudden infant death syndrome. Lancet 337: 1244-1247, 1991.

20. Chang SH and Miller NR: The incidence of vision loss due to perioperative ischemic optic neuropathy associated with spine surgery: The Johns Hopkins Hospital Experience. Spine (Phila Pa 1976) 30: 1299-1302, 2005.

21. Arndt JO, Bömer W, Krauth J and Marguardt B: Incidence and time course of cardiovascular side effects during spinal anesthesia after prophylactic administration of intravenous fluids or vasoconstrictors. Anesth Analg 87: 347-354, 1998.

22. Ngan Kee WD, Khaw KS and Ng FF: Prevention of hypotension during spinal anesthesia for cesarean delivery: An effective technique using combination phenylephrine infusion and crystalloid cohydration. Anesthesiology 103: 744-750, 2005.

23. Sookplung P, Siriussawakul A, Malakouti A, Sharma D, Wang J, Souter MJ, Chesnut RM and Vavilala MS: Vasopressor use and effect on blood pressure after severe adult traumatic brain injury. Neurocrit Care 15: 46-54, 2011.

24. Thiele RH, Nemergut EC and Lynch C III: The clinical implications of isolated alpha(1) adrenergic stimulation. Anesth Analg 113: 297-304, 2011.

25. Thiele RH, Nemergut EC and Lynch C III: The physiologic implications of isolated alpha(1) adrenergic stimulation. Anesth Analg 113: 284-296, 2011.

26. Gelman S: Venous function and central venous pressure: A physiologic story. Anesthesiology 108: 735-748, 2008.
27. Cannesson M, Jian Z, Chen G, Vu TQ and Hatib F: Effects of phenylephrine on cardiac output and venous return depend on the position of the heart on the Frank-Starling relationship. J Appl Physiol 113: 281-289, 2012.

28. Cannesson M, Jian Z, Chen G, Vu TQ and Hatib F: Effects of phenylephrine on cardiac output and venous return depend on the position of the heart on the Frank-Starling relationship. J Appl Physiol 113: 281-289, 1985.

29. Saravanan S, Kocarev M, Wilson RC, Watkins E, Columb MO and Lyons G: Equivalent dose of ephedrine and phenylephrine in the prevention of post-spinal hypotension in Caesarean section. Br J Anaesth 96: 95-99, 2006.

30. Goyagi T, Tanaka M and Nishikawa T: Oral clonidine premedication enhances the pressor response to ephedrine during spinal anesthesia. Anesth Analg 87: 1336-1339, 1998.

31. Hayakawa-Fujii Y, Iida H and Dohi S: Propofol anesthesia enhances pressor response to ephedrine in patients given clonidine. Anesth Analg 89: 37-41, 1999.

32. Kanaya N, Satoh H, Seki S, Nakayama M and Namiki A: Propofol anesthesia enhances the pressor response to intravenous ephedrine. Anesth Analg 94: 1207-1211, 2002.

33. Nishikawa T, Kimura T, Taguchi $\mathrm{N}$ and Dohi S: Oral clonidine preanesthetic medication augments the pressor responses to intravenous ephedrine in awake or anesthetized patients. Anesthesiology 74: 705-710, 1991.

34. Behnia M, Powell S, Fallen L, Tamaddon H and Behnia M: Correlation of stroke volume measurement between Sonosite portable echocardiogram and Edwards Flotrac sensor-Vigileo monitor in an intensive care unit. Clin Med Insights Circ Respir Pulm Med 7: 45-51, 2013.

35. Camporota $\mathrm{L}$ and Beale R: Pitfalls in haemodynamic monitoring based on the arterial pressure waveform. Crit Care 14: 124, 2010.

36. Meng L, Tran NP, Alexander BS, Laning K, Chen G, Kain ZN and Cannesson M: The impact of phenylephrine, ephedrine and increased preload on third-generation Vigileo-FloTrac and esophageal doppler cardiac output measurements. Anesth Analg 113: 751-757, 2011. 